\title{
Striatal Activity during Intentional Switching Depends on Pattern Stability
}

\author{
Cinzia De Luca, ${ }^{1,2}$ Kelly J. Jantzen, ${ }^{3,4}$ Silvia Comani, ${ }^{1,2,5}$ Maurizio Bertollo, ${ }^{2,6}$ and J. A. Scott Kelso ${ }^{4,7}$ \\ ${ }^{1}$ Department of Clinical Sciences and Bioimaging and ${ }^{2}$ Behavioral Imaging and Neural Dynamics Center, Gabriele d'Annunzio University, 66013 Chieti, \\ Italy, ${ }^{3}$ Western Washington University, Bellingham, Washington 98225, ${ }^{4}$ Center for Complex Systems and Brain Sciences, Florida Atlantic University, \\ Boca Raton, Florida 33431, ${ }^{5}$ Casa di Cura Privata Villa Serena, Città S. Angelo 65013 Pescara, Italy, ${ }^{6}$ Department of Basic and Applied Medical Sciences, \\ Gabriele d'Annunzio University, Chieti 66013, Italy, and 7Intelligent Systems Research Centre, University of Ulster, Magee Campus, L’Derry BT48 7JL, \\ N. Ireland
}

The theoretical framework of coordination dynamics posits complementary neural mechanisms to maintain complex behavioral patterns under circumstances that may render them unstable and to voluntarily switch between behaviors if changing internal or external conditions so demand. A candidate neural structure known to play a role in both the selection and maintenance of intentional behavior is the basal ganglia. Here, we use functional magnetic resonance imaging to explore the role of basal ganglia in intentional switching between bimanual coordination patterns that are known to differ in their stability as a function of movement rate. Key measures of pattern dynamics and switching were used to map behavior onto the associated neural circuitry to determine the relation between specific behavioral variables and activated brain areas. Results show that putamen activity is highly sensitive to pattern stability: greater activity was observed in bilateral putamen when subjects were required to switch from a more to a less stable pattern than vice versa. Since putamen activity correlated with pattern stability both before and during the switching process, its role may be to select desired actions and inhibit competing ones through parametric modulation of the intrinsic dynamics. Though compatible with recent computational models of basal ganglia function, our results further suggest that pattern stability determines how the basal ganglia efficiently and successfully select among response alternatives.

\section{Introduction}

The role of the basal ganglia (BG) in the selection of intentional actions is supported by empirical (Cools, 1980; Kropotov and Etlinger, 1999; Troyer et al., 2004; Cools et al., 2007; Seger, 2008) and theoretical (Mink, 1996; Humphries and Gurney, 2000; Gurney et al., 2001; Humphries et al., 2006; Girard et al., 2005; Ponzi, 2008) contributions. However, how the BG functionally operates in action selection is still a matter of debate. It has been hypothesized that BG may enact a broad inhibition of all patterns coupled with a focused disinhibition of the desired one (Mink, 1996) depending on intention, environmental demand, and the intrinsic salience of actions (Bar-Gad et al., 2003; Gurney et al., 2004). Thus, BG involvement during action selection and switching may be moderated by key variables that capture the interaction between behavior and environment.

Here, we consider action selection within the framework of coordination dynamics (Kelso, 2009). A central tenet of coordination dynamics is that switching between patterns depends

\footnotetext{
Received June 8, 2009; revised Dec. 2, 2009; accepted Dec. 15, 2009.

This research was supported by National Institutes of Health Grants NS48229 and MH080838 and the Chaire Pierre de Fermat to J.A.S.K. and by Progetto Operativo Regionale Abruzzo 2000-2006 C3/IC4E Grant to C.D.L. We thank our colleague Dr. F. Steinberg for making available the facilities at University MRI, Boca Raton, FL.

Correspondence should be addressed to Dr. Cinzia De Luca, Department of Clinical Science and Bioimaging and Behavioral Imaging and Neural Dynamics Center, Gabriele d'Annunzio University, Via Dei Vestini 33, 66013 Chieti, Italy. E-mail: c.deluca@unich.it.

D0I:10.1523/JNEUROSCI.2673-09.2010

Copyright $\odot 2010$ the authors $\quad 0270-6474 / 10 / 303167-08 \$ 15.00 / 0$
}

upon their stability (Kelso, 1995; Swinnen, 2002; Park and Turvey, 2008), as assessed in behavioral and neuroimaging studies using quantitative measures of stability such as relaxation time, switching time, and variability (Kelso et al., 1992; Mayville et al., 1999; Daffertshofer et al., 2000; Fuchs et al., 2000a,b; Meyer-Lindenberg et al., 2002; Aramaki et al., 2006; Jantzen et al., 2009). In coordination dynamics, manipulation of key control parameters results in a loss of stability and spontaneous switching to patterns that better meet current demands. The extension to intentional pattern selection and change is supported by behavioral evidence and theoretical modeling showing (1) that intention parameterizes the dynamics and (2) that intentional switching from more to less stable patterns takes longer than the reverse (Kelso et al., 1988; Schöner and Kelso, 1988; Scholz and Kelso, 1990; Lee et al., 1996). One interpretation of the foregoing holds that the longer switching time is required to destabilize the more stable pattern and simultaneously stabilize the less stable one, a task we hypothesize is mediated by BG and its connected cortical circuitry. Accordingly, transient disruption of major cortical BG targets [supplementary motor area (SMA) and premotor cortex (PMC)] may induce spontaneous switching from less to more stable movement patterns but not the reverse (Meyer-Lindenberg et al., 2002). These results suggest that intention exerted through BG circuits both acts upon and is constrained by the intrinsic dynamics of coordination.

In the present study, we use functional magnetic resonance imaging in conjunction with an established bimanual coordina- 
tion paradigm to investigate the role of the BG in mediating the interaction between pattern stability and intentional switching. By defining and manipulating pattern stability (Kelso, 1984; Kelso et al., 1988), we were able to map the coordination dynamics onto the underlying neural circuitry (Jantzen and Kelso, 2007). This step allowed us to test the dynamic hypothesis that the active destabilization of a stable pattern concurrent with the selection and stabilization of a less stable one places greater demands on the BG and related neural circuitry than vice versa.

\section{Materials and Methods}

Subjects. A total of 12 healthy normal right handed subjects ( 8 male, 4 female; age range: $28 \pm 6$ years), participated in this study. All participants gave written informed consent before participating in the experiment, according to the guidelines approved by the Internal Review Board of Florida Atlantic University and conforming to the principles of the Declaration of Helsinki.

Task and experimental procedure. Participants coordinated flexion and extension movements of their left and right index fingers in either anti-phase (AP) or in-phase (IP) mode. The AP mode (Fig. $1 A$ ) was formed by alternating finger flexion/extension in a parallel pattern, whereas the IP mode (Fig. 1B) was executed by simultaneous flexion/extension of the fingers, resulting in a mirror symmetric pattern. On each trial, participants performed coordinated bimanual movements paced by an auditory stimulus presented at $1.52,2.0$, or 2.5 $\mathrm{Hz}$ over MR-compatible headphones. Oscillation frequencies were selected to avoid the critical values at which spontaneous transitions between coordination patterns are known to occur (Kelso, 1984; Scholz and Kelso, 1990). Before each trial, a visual instruction indicated which pattern to execute. Once on each trial, a change in metronome pitch prompted participants to intentionally switch from the initial to the alternative pattern, that is, from IP to AP or vice versa. The combination of oscillation frequency (or coordination rate) and pattern resulted in six trial types that systematically probed neural mechanisms of intentional switching between patterns of differing stability. Trial types were pseudorandomized in four sessions each consisting of 18 trials. Between each trial, subjects remained still and viewed a centrally presented fixation cross. Each trial lasted $24 \mathrm{~s}$ and was followed by a rest period of $14 \mathrm{~s}$. The switch was prompted approximately halfway into each trial. The relative position of the fingers was recorded by changes in pressure within small air-filled pillows that were converted to a voltage, sampled at $1 \mathrm{kHz}$, and stored together with stimulus and MRI magnet timing signals for off-line analysis.

Image acquisition. Functional BOLD activity was acquired using an echo-planar pulse sequence $\left(\mathrm{TR}=2 \mathrm{~s}, \mathrm{TE}=35 \mathrm{~ms}, \mathrm{FA}=90^{\circ}\right.$,

\section{A AP pattern}

\section{AP to IP}
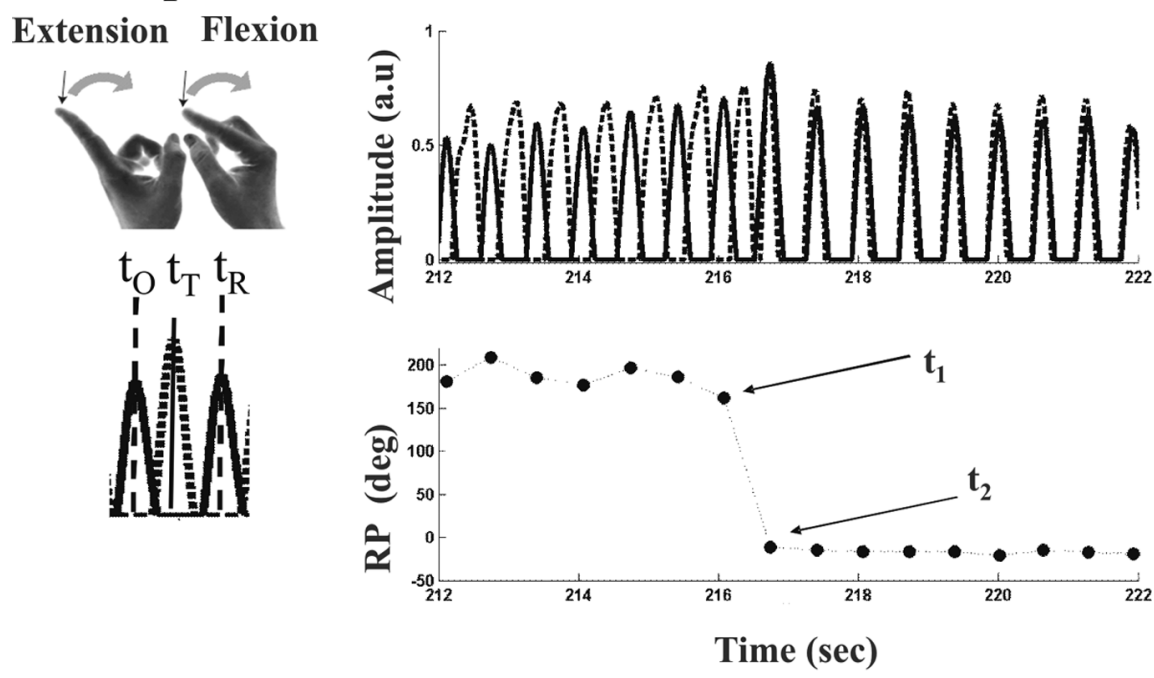

B IP pattern D IP to AP
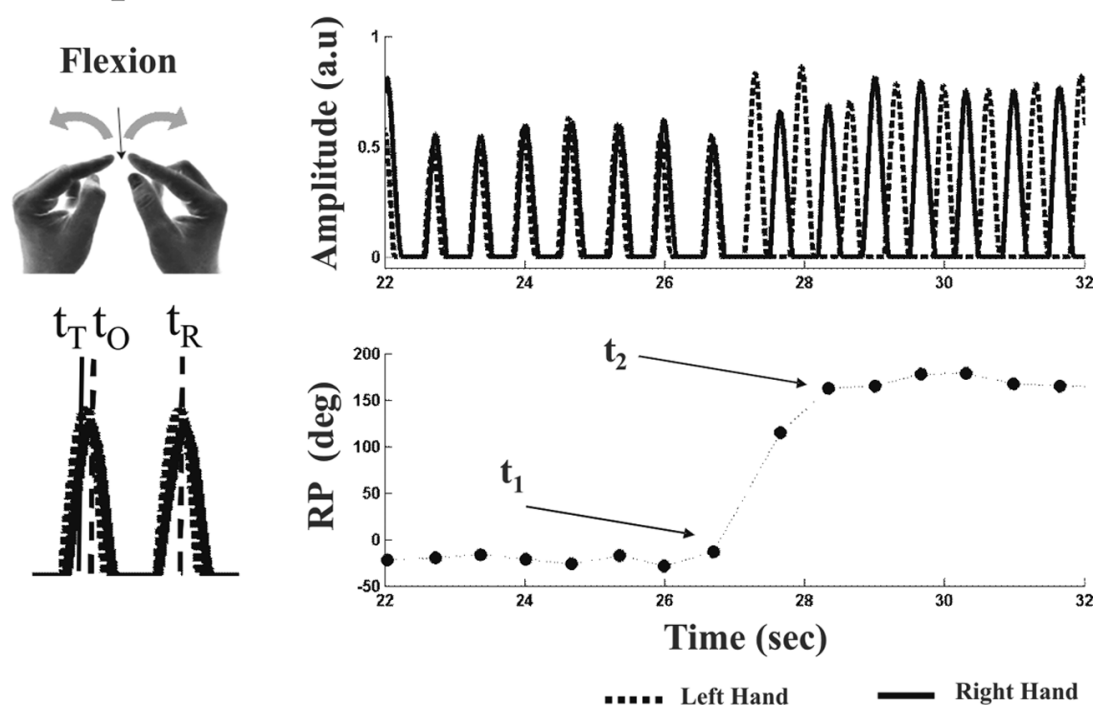

Figure 1. $\quad \boldsymbol{A}, \boldsymbol{B}$, Schematic representation of the AP $(\boldsymbol{A})$ and IP $(\boldsymbol{B})$ patterns of coordination. Time points $t_{0}$ and $t_{\mathrm{R}}$ in $\boldsymbol{A}$ and $\boldsymbol{B}$ correspond to consecutive peaks of flexion of the right index finger, whereas $t_{\mathrm{T}}$ corresponds to the peak flexion of the left index finger, closest in time to $t_{\mathrm{T}} \mathbf{C}, \boldsymbol{D}$, Behavioral responses and the associated relative phase estimate for the AP to IP condition $(\boldsymbol{C})$ and the IP to AP condition (D). Time points $t_{1}$ and $t_{2}$ represent the onset and the offset of intentional switching, respectively.
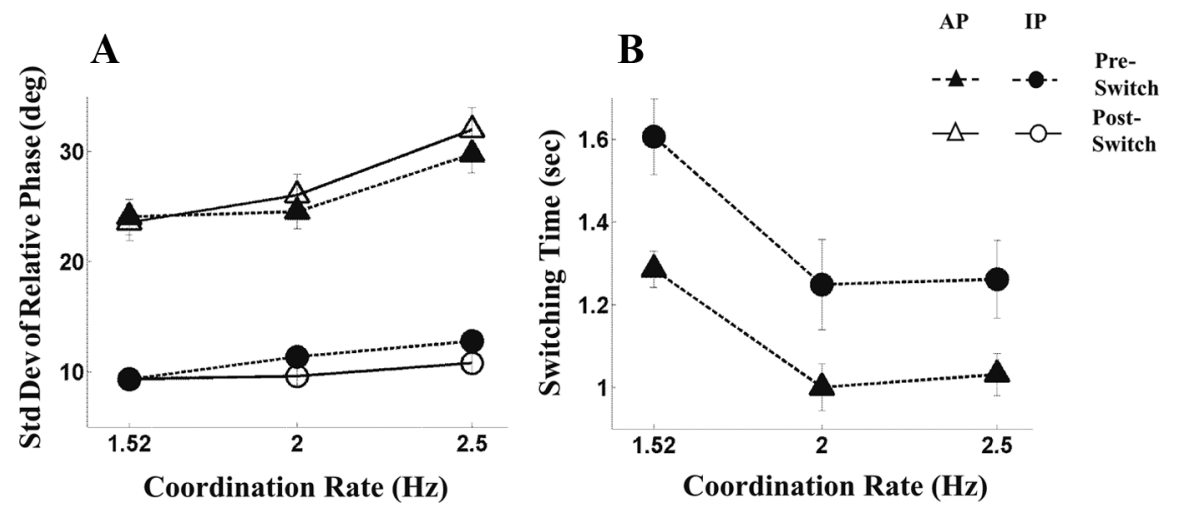

Figure 2. Behavioral results. $A$, Pattern variability across conditions is shown by the SD of the relative phase plotted as a function of coordination rate. Open circles (triangles) refer to grand averages of the IP (AP) pattern, measured during the preswitch epochs. Solid symbols refer to the averages during the postswitch epochs. $\boldsymbol{B}$, Switching time is plotted as a function of coordination rate. Circles (triangles) refer to grand averages of the switching time during an intentional switch from IP to AP pattern (from AP to IP pattern). Error bars indicate SEM. 
Table 1. Center of mass location of clusters showing a significant $(p<0.05)$ correlation between model BOLD function (regressor) and actual BOLD, during the preswitch epoch

\begin{tabular}{|c|c|c|c|c|c|c|c|}
\hline \multirow{2}{*}{$\frac{\text { Regressor }}{\text { Coordination rate }}$} & \multirow{2}{*}{$\begin{array}{l}\text { Side } \\
R\end{array}$} & \multirow{2}{*}{$\begin{array}{l}\text { Cluster size } \\
9088\end{array}$} & \multicolumn{3}{|c|}{ MNI brain $x, y, z$ coordinates (mm) } & \multirow{2}{*}{$\begin{array}{l}\text { Anatomical description } \\
\text { Postcentral gyrus }\end{array}$} & \multirow{2}{*}{$\frac{B A}{3}$} \\
\hline & & & 38 & -33 & 53 & & \\
\hline & $\mathrm{L}$ & 6080 & -24 & -33 & 50 & Precentral gyrus & 3 \\
\hline & $\mathrm{R}$ & 3264 & 40 & -27 & 11 & Transverse temporal gyrus & 41 \\
\hline & $\mathrm{R}$ & 1728 & 13 & -24 & 55 & Medial frontal gyrus & 6 \\
\hline & $\mathrm{R}$ & 896 & 54 & -14 & 8 & Superior temporal gyrus & \\
\hline & $\mathrm{L}$ & 896 & -41 & -29 & 9 & Transverse temporal gyrus & \\
\hline \multirow[t]{6}{*}{ Preswitch pattern stability } & $\mathrm{R}$ & 8448 & 2 & -13 & 60 & Medial frontal gyrus & 6 \\
\hline & $\mathrm{L}$ & 2560 & -44 & -5 & 36 & Precentral gyrus & 6 \\
\hline & $\mathrm{L}$ & 1600 & -46 & 1 & 27 & Inferior frontal gyrus & \\
\hline & $\mathrm{R}$ & 1408 & 48 & -54 & -2 & Middle temporal gyrus & \\
\hline & $\mathrm{L}$ & 1344 & -42 & -59 & 3 & Middle temporal gyrus & \\
\hline & $\mathrm{L}$ & 832 & -46 & -16 & 47 & Precentral gyrus & 4 \\
\hline
\end{tabular}

Table 2. Center of mass location of clusters showing a significant $(p<0.05)$ correlation between model BOLD function (regressor) and actual BOLD, during the switch epoch

\begin{tabular}{|c|c|c|c|c|c|c|c|}
\hline \multirow{2}{*}{$\begin{array}{l}\text { Regressor } \\
\text { Preswitch pattern stability }\end{array}$} & \multirow{2}{*}{$\frac{\text { Side }}{\mathrm{R}}$} & \multirow{2}{*}{$\begin{array}{l}\text { Cluster size } \\
4096\end{array}$} & \multicolumn{3}{|c|}{ MNI brain $x, y, z$ coordinates (mm) } & \multirow{2}{*}{$\begin{array}{l}\text { Anatomical description } \\
\text { Putamen }\end{array}$} & \multirow[t]{2}{*}{ BA } \\
\hline & & & 27 & 1 & 7 & & \\
\hline & $\mathrm{L}$ & 3200 & -20 & 1 & 7 & Putamen & \\
\hline & $\mathrm{R}$ & 1920 & 2 & -9 & 59 & Medial frontal gyrus & 6 \\
\hline Switching time duration & $\mathrm{L}$ & 3136 & -1 & 0 & 49 & Cingulate lobe & \\
\hline
\end{tabular}

Table 3. Center of mass location of clusters showing a significant $(p<0.05)$ correlation between model BOLD function (regressor; coordination rate) and actual BOLD, during the postswitch epoch

\begin{tabular}{|c|c|c|c|c|c|c|}
\hline \multirow{2}{*}{ Side } & \multirow{2}{*}{$\begin{array}{l}\text { Cluster size } \\
12,608\end{array}$} & \multicolumn{3}{|c|}{ MNI brain $x, y, z$ coordinates (mm) } & \multirow{2}{*}{$\begin{array}{l}\text { Anatomical description } \\
\text { Precentral gyrus }\end{array}$} & \multirow{2}{*}{$\frac{B A}{4}$} \\
\hline & & -28 & -31 & 53 & & \\
\hline $\mathrm{R}$ & 11,776 & 39 & -29 & 50 & Postcentral gyrus & 40 \\
\hline $\mathrm{R}$ & 3136 & 14 & -21 & 54 & Medial frontal gyrus & 6 \\
\hline R & 2496 & 46 & -9 & 9 & Insula & \\
\hline L & 2368 & -5 & -58 & -20 & Cerebellum-declive & \\
\hline L & 2368 & -40 & -22 & 9 & Insula & \\
\hline R & 576 & 31 & -7 & 31 & Precentral gyrus & 4 \\
\hline
\end{tabular}

FOV $=240 \mathrm{~mm}, 64 \times 64$ matrix) on a $3 \mathrm{~T}$ General Electric MRI (GE Medical Systems). Each volume covered the entire brain and consisted of thirty slices, each $5 \mathrm{~mm}$ thick with an in-plane resolution of $3.75 \times 3.75$ $\mathrm{mm}$. For each subject, high-resolution spoiled gradient-recalled at steady-state images were acquired at the end of the experiment. These images, which have the same in-plane alignment and thickness of the EPI images but better within-plane resolution, were collected as anatomical reference for the coregistration of the functional images.

Behavioral analysis. Behavioral performance was quantified by calculation of the central tendency and variability in relative phase (RP), a key collective variable known to characterize patterns of coordination (Kelso, 2009). As shown in Figure 1, A and B, RP was calculated on each movement cycle as the temporal difference between time points associated with the maximal flexion of the left finger $\left(t_{\mathrm{T}}\right)$ and the maximal flexion of the right one $\left(t_{\mathrm{O}}\right)$, divided by the interval between maximal flexion of the right finger on the current $\left(t_{\mathrm{O}}\right)$ and subsequent cycle $\left(t_{\mathrm{R}}\right)$. The resulting normalized differences between the fingers were projected onto the unit circle within the range $-180^{\circ}$ to $180^{\circ}$, where a positive (negative) value indicated that the right finger was leading (lagging) the left finger. Perfect AP produces a RP of $\pm 180^{\circ}$, whereas perfect IP performance yields a relative phase of $0^{\circ}$ (Fig. $1 B, C$ ). RP thus provides a discrete measure of the coordination pattern. The SD of the RP (RPSD) computed across each trial block provides an operational estimate of a pattern's stability (proportional to the inverse of pattern variability).

The switching time, or the time interval required to discontinue the ongoing pattern and adopt a new one, is a key behavioral measure of the interaction between intention and intrinsic dynamics (Kelso et al., 1988; Scholz and Kelso, 1990). Operationally, switching time was defined as the time difference between the last movement cycle meeting the RP crite- rion for the preswitch pattern (Fig. $1, t_{1}$ ) and the first cycle meeting the $\mathrm{RP}$ criterion for the postswitch pattern (Fig. 1, $t_{2}$ ). The aforementioned criterion was defined operationally as \pm 1 SD of the preswitch and postswitch mean RP. The onset of switching was defined as the final movement cycle before the RP measure deviated from the preswitch pattern criteria on at least three successive cycles. Similarly, switching offset was defined as the first of three consecutive cycles that met criteria for the postswitch pattern. Switching time was defined as the duration between switch onset and offset. Each trial was divided into preswitch (before switch onset), postswitch (following switch offset), and switch (between switch onset and offset) epochs.

Neuroimaging analysis. BOLD data were preprocessed and analyzed using AFNI (Cox, 1996; Cox and Hyde, 1997). After correcting for slice timing and head motion, the amplitude of each dataset was normalized, spatially filtered using an isotropic Gaussian blur with full width at half maximum of $8 \mathrm{~mm}$, and temporally filtered using a low-pass filter with a cutoff frequency of $0.2 \mathrm{~Hz}$. High-resolution images were registered using FSL (Jenkinson and Smith, 2001) to a canonical brain transformed into the coordinate space of Talairach and Tournoux (1988). The same transformation was applied to the functional images before concatenating the different sessions into a single BOLD time series.

Two sets of analyses were performed at the single-subject level. The first estimated the magnitude of neural activity associated with each coordination rate and pattern. The BOLD signal related to the six experimental conditions was modeled separately for the preswitch, switch, and postswitch epochs, resulting in 18 covariates [(AP, IP pattern $) \times(1.52,2.00$, $2.50 \mathrm{~Hz}$ coordination rate $) \times$ (preswitch, switch, postswitch epochs)], each generated by convolving a canonical hemodynamic response function (HRF) with vectors containing the appropriate functional task response 
and epoch timing. Preswitch BOLD signals were modeled from the onset of the trial to the start of the switch (Fig. 1, $t_{1}$ ). Postswitch BOLD was modeled from the switch offset (Fig. 1, $t_{2}$ ) to trial offset, and the switch epoch was modeled in the interval $t_{1}-t_{2}$.

The second examined the predicted relationship between coordination parameters, switching and brain function. Previous work identified two distributed cortical circuits associated with coordination rate and pattern stability (Jantzen et al., 2009). Here, we adopt a similar analysis to investigate how coordination rate and stability relate to neural activity during intentional switching. To this end, the BOLD signal of each epoch (preswitch, postswitch, and switch) was modeled by covariates representing (1) coordination rate, (2) stability of the preswitch pattern (measured by RPSD), and (3) switching time. Additional covariates of no interest modeled offset, drift, and main effects of movement as part of a baseline model.

A random-effects analysis was performed at the group level by voxelwise one-sample $t$ tests applied to each parameter estimate. Multiple comparisons were corrected at $\alpha<0.05$ by applying a combined statistical and cluster size threshold in which clusters of active voxels were considered significant if they exceeded a per voxel threshold of $p<0.001$ and were at least $576 \mathrm{~mm}^{3}$ in size. The estimated statistical and geometrical significance of activation clusters were computed using Monte Carlo simulation (AFNI's AlphaSim).

\section{Results}

\section{Behavioral data}

All subjects successfully performed the assigned pattern of coordination across all rates and in the absence of spontaneous transitions. The RPSD, our estimate of pattern stability, was greater during AP than IP coordination for both preswitch and postswitch epochs (Fig. 2 A). A pattern (IP, AP) $\times$ rate $(1.52,2.0,2.5$ $\mathrm{Hz}) \times$ epoch (preswitch, switch, postswitch) ANOVA performed on RPSD revealed a significant main effect of rate $\left(F_{(2,156)}=13.63\right.$, $p<0.01)$, main effect of pattern $\left(F_{(2,156)}=440.15, p<0.01\right)$, and pattern $\times$ rate interaction $\left(F_{(2,156)}=3.71, p<0.01\right)$. The latter interaction results because the decrease in stability (increase in variability) with increasing coordination rate is greater for the $\mathrm{AP}$ than the IP pattern.

A similar ANOVA performed on switching times revealed a significant main effect of rate $\left(F_{(2,78)}=10.55, p<0.001\right)$ and pattern $\left(F_{(1,78)}=17.45, p<0.001\right)$, with no interaction. Switching time is significantly longer when switching from a more stable (IP) to a less stable (AP) pattern (Fig. 2B). Accordingly, we observed that the switching time shortened with decreasing stability (increasing variability) of the preswitch pattern.

\section{Functional neuroimaging}

Brain regions in which the BOLD signal during the preswitch, switch, and postswitch epochs was significantly correlated with coordination rate, stability of the preswitch pattern, and switching time duration are summarized in Table 1, Table 2, and Table 3 , respectively.

\section{B Stability dependent}

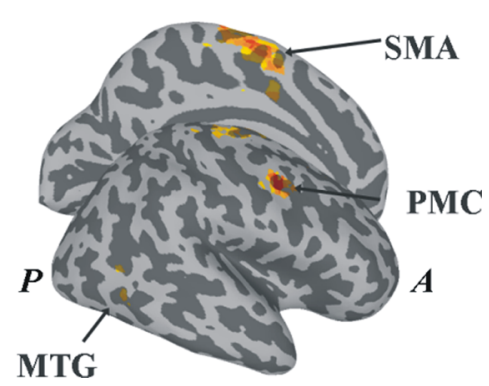

MTG

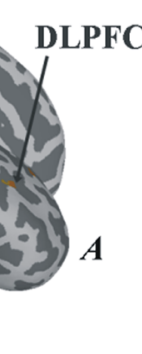

Figure 3. $A, B$, Brain regions demonstrating a significant relationship between $B O L D$ amplitude and coordination rate in the preswitch epoch $(\boldsymbol{A})$ and pattern stability $(\boldsymbol{B})$ regardless of coordination pattern (IP or AP). Since preswitch stability was estimated as $1 /$ RPSD, a positive correlation means an increase in BOLD activity as the pattern being executed becomes more variable (less stable). A, anterior; $P$, posterior.
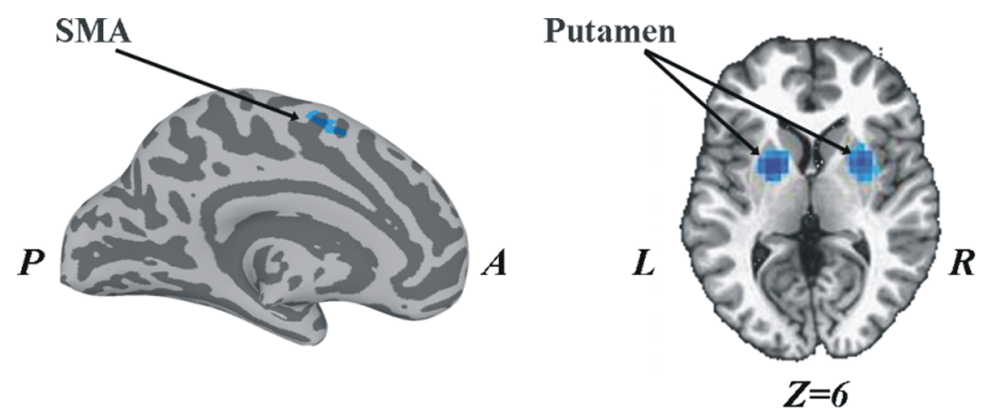

Figure 4. Brain regions in which BOLD activity during intentional switching was correlated with preswitch pattern stability. A, anterior; $P$, posterior; $L$, left; $R$, right.

\section{Preswitch epoch}

Brain regions in which preswitch BOLD changes correlated significantly with coordination rate and initial pattern stability are shown in Figure 3. Rate-dependent BOLD increases were observed in bilateral primary sensorimotor cortex, SMA, and STG (Fig. 3A). BOLD activity was positively related to pattern variability in premotor regions including SMA and dorsal PMC, as well as in bilateral middle temporal gyrus (MTG) (Fig. 3B).

\section{Switch epoch}

Switch-related BOLD activity was not correlated with coordination rate. In contrast, a negative correlation was observed between BOLD activity and initial pattern stability in pre-SMA and bilateral putamen (Fig. 4). Here, the putamen of the striatum (the input structures of the BG) showed stability-related activity only during switching but not during the preswitch or postswitch epochs, suggesting that stability influences putamen function only during intentional switching.

To further illustrate the relationship between frontostriatal activity and pattern stability, we plotted the normalized BOLD response, derived by normalizing the $\beta$ weight of each subject across conditions, from the peak voxel in SMA and left putamen during the preswitch (Fig. 5A), switch (Fig. 5B), and postswitch (Fig. 5C) epochs together with preswitch pattern variability and stability (1/RPSD). During preswitch (Fig. $5 A$ ) and postswitch (Fig. 5C) epochs, SMA but not putamen activity follows the pattern of variability, being more active when patterns are more variable (or less stable). During switching, inhibition of a more stable pattern in favor of a less stable pattern (IP-AP conditions), required more activity in SMA and Putamen than switching in the reverse (AP-IP) direction. Importantly, the switching-related 


\section{A Pre-Switch Epoch}

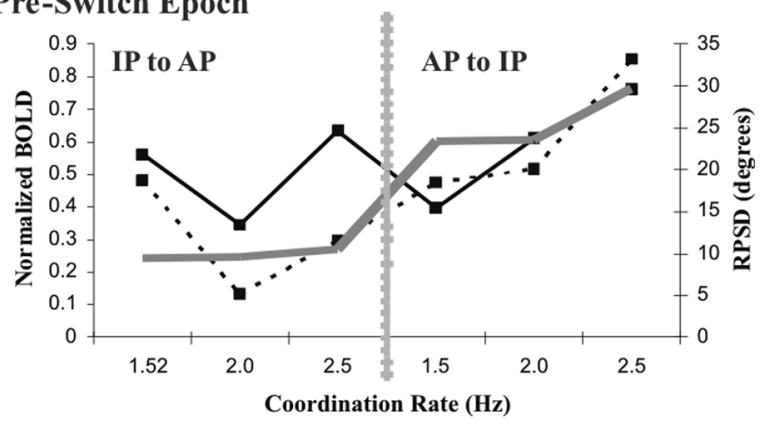

B Switch Epoch

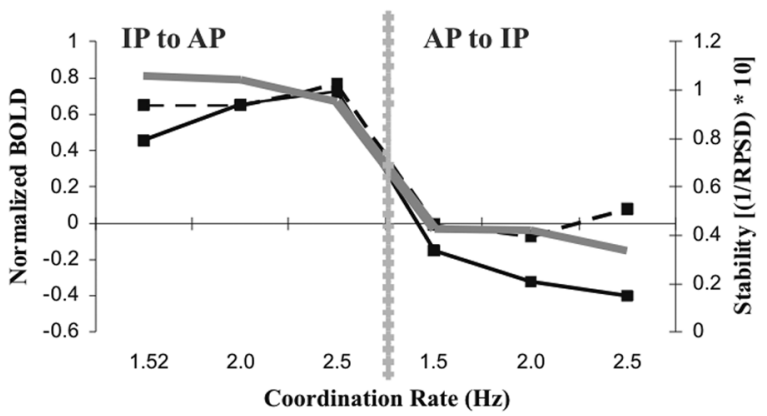

\section{Post-Switch Epoch}

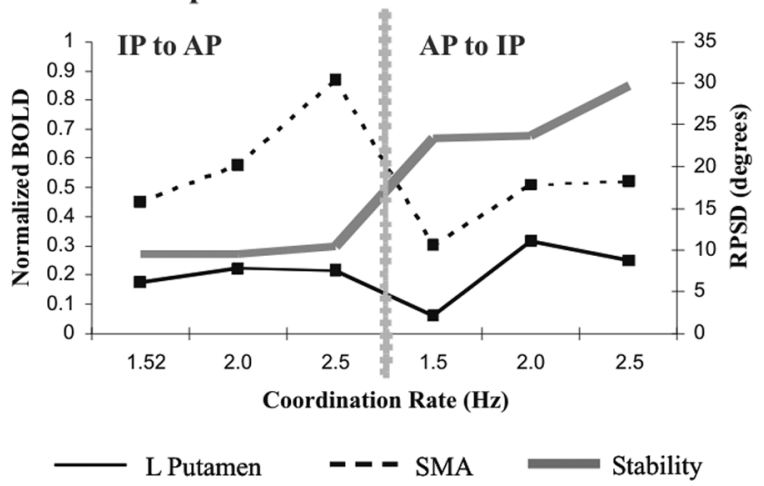

Figure 5. $\quad \boldsymbol{A}-\boldsymbol{C}$, Normalized BOLD activity in SMA (dashed line) and in left putamen (solid line) during the preswitch $(\boldsymbol{A})$, switch $(\boldsymbol{B})$, and postswitch $(\boldsymbol{C})$ epochs. The left of each panel shows the three coordination rates when switching from the in-phase to the anti-phase (IPAP) pattern, whereas the right of each plot shows the BOLD amplitude when switching from the anti-phase to in-phase pattern (AP-IP). In $A$ and $C$, the BOLD activity is plotted together with the $S D$ of preswitch relative phase (solid gray line) to show the relationship between BOLD and pattern variability. In $A$, activity in SMA (but not the putamen) increases with increasing pattern variability (RSPD). In C, SMA activity shows the opposite correlation indicating activity is now related to postswitch variability. Again there is no relationship of putamen activity to stability. In $B, B O L D$ amplitude is plotted together with preswitch stability (1/RSPD) to show that activity in both SMA and putamen scale with the stability of the pattern, being greater when switching from a more stable to a less stable pattern and vice versa.

BOLD activity in SMA and putamen closely followed the stability of the preswitch pattern. Note that Figure $5 B$ plots stability as the inverse of variability to better demonstrate this key relationship. Thus, greater stability indicated by the gray line in Figure $5 B$ signals less variability in the preswitch coordination pattern. Thus, more activity in a frontostriatal circuit is required when switching from a more to less stable pattern.

A switching time covariate investigated the possibility that BOLD activity during switching was a function of switching time as opposed to stability per se. Longer switching times were associated with greater BOLD activity in bilateral cingulate cortex area (Fig. 6), a region that did not overlap with areas demonstrat-

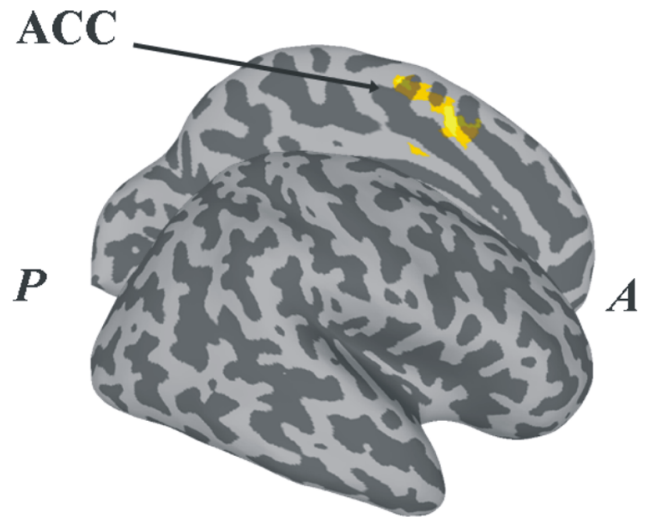

Figure 6. Brain regions in which BOLD activity is significantly correlated with switching time. $A$, anterior; $P$, posterior.

ing either rate- or stability-related activity. Lesions in the cingulate cortex are known to disrupt the spatial and temporal organization of bimanual coordination (Kennerley et al., 2002), attesting to its general role in the organization of movement (Shima and Tanji, 2000). Moreover, CMA has been observed during spontaneous transitions (Aramaki et al., 2006) and has been attributed a role in the detection of stimulus-response conflict (Paus, 2001). The present correlation between CMA and switching time may be interpreted as CMA involvement during the resolution of a response conflict that persists over the switching duration. Importantly, this analysis demonstrates that activity in putamen and SMA during the switch cannot be attributed only to an alteration in the switching time.

\section{Postswitch epoch}

Brain regions in which postswitch BOLD changes correlated significantly with coordination rate and preswitch pattern stability are shown in Figure 7. Postswitch-related BOLD activity was not correlated neither with preswitch pattern stability or switching time duration. In contrast, BOLD activity was positively related to coordination rate in bilateral precentral gyrus, right SMA, bilateral insula, and left cerebellum (declive).

\section{Discussion}

Using the well defined experimental model system of bimanual coordination as a window, we aimed to uncover the neural mechanisms of intentional switching between behavioral patterns, with particular attention to the role played by the BG. Coordination Dynamics provided the conceptual and methodological framework for (1) mapping stability of coordination patterns onto neural circuits and (2) investigating how brain areas related to pattern switching are influenced both by one's intention and the intrinsic dynamics of the behavioral patterns one is switching between. A remarkable result is that the role of putamen in pattern selection and switching is directly connected to the stability of the preceding and ensuing pattern as quantified by the collective variable, relative phase.

Our demonstration of a dissociation between rate and stability-dependent patterns of BOLD activity during preswitch coordination is in keeping with previous findings (MeyerLindenberg et al., 2002; Debaere et al., 2004; Jantzen and Kelso, 2007; Jantzen et al., 2009). The rate-dependent activity in auditory and motor cortices likely reflects sensitivity to parameters of individual sensorimotor components such as the number of movements (Sadato et al., 1996), muscle force (Thickbroom et al., 1998), hand speed (Jerbi et al., 2007) and the rate of auditory 
stimulation (Price et al., 1992; Binder et al., 1994). In contrast, premotor regions respond to changes in parameters that specify the coordination state of the system as measured by quantities such as relative phase and its stability (Kelso et al., 1992; Meyer-Lindenberg et al., 2002; Jantzen and Kelso, 2007; Jantzen et al., 2009).

The present behavioral results corroborate previous empirical observations and theoretical analysis that demonstrates an interaction between intentional switching and the intrinsic dynamics of coordination (Kelso et al., 1988; Schöner and Kelso, 1988; Scholz and Kelso, 1990; Lee et al., 1996). Like this body of work, we found that the time necessary to switch between patterns was modulated by pattern stability; switching time was longer when transitioning from a more to a less stable pattern than vice versa. Moreover, switching time monotonically decreased with decreasing pattern stability and increasing coordination rate. Within a more general equation of motion that accurately models key coordination phenomena, Kelso et al. (1988) and Scholz and Kelso (1990) represented intention as a single parameter that simultaneously increases the stability of the desired pattern and decreases the stability of the ongoing pattern. When imposed on the natural or intrinsic dynamics of the system, the resulting mathematical description at both coordinated and component levels of description accurately predicts the required switching time (Schöner and Kelso, 1988). In principle, the complementary and simultaneous processes of inhibition/ selection suggested in the foregoing work are compatible with proposed models for the role of BG in mediating pattern selection and switching. That is, the role of intention is to destabilize the ongoing pattern, cause a switch, and simultaneously stabilize the new, intended pattern.

Although the neural mechanisms underlying intentional switching and their connection to pattern stability have not previously been directly explored, a number of studies have provided evidence that supports our current findings. An important fMRI and PET study by Meyer-Lindenberg et al. (2002), for example, investigated spontaneous switching between bimanual patterns known to differ in stability, revealing the importance of frontal motor areas in maintaining pattern stability. Kraft et al. (2007) reported enhanced BG activity when initiating anti-phase hand movements but did not show any difference in putamen activity between mirror and parallel patterns during continuous execution, a finding in keeping with the present result that striatum is involved in selecting and switching between patterns in a stability-dependent manner but not in pattern execution per se. Furthermore, Toxopeus et al. (2007) showed BG and SMA activity in response to both movement initiation and inhibition, and Li et al. (2008) detected putamen activity correlated with oscillation frequency in a study on motor response inhibition during a stop signal task. Yehene et al. (2008) further reported deficits in task switching in patients with focal lesions within frontal-subcortical circuits. In the present work, the pattern of putamen activation associated with intentional switching corroborates previous observations attributing to the $\mathrm{BG}$ a role in the process of selection of desired actions and inhibition of competing ones (Mink, 1996; Kropotov and Etlinger, 1999; Troyer et al., 2004; Cools et al., 2007; Seger, 2008). However, a key new finding here is that putamen activity during intentional switching is correlated with pattern stability and that putamen activation is sensitive to the intrinsic coordination dynamics of the selection/inhibition process.

In a previous imaging study on spontaneous transitions between coordination patterns, in which pattern selection is determined by the intrinsic dynamics alone without any influence of intention, BG activity was greater for AP than IP coordination during pattern maintenance, but not during spontaneous transitions (Aramaki et al., 2006). Based on our interpretation of the $B G$ 's role in intentional pattern selection, recruitment of the BG would not be expected during spontaneous transitions. The latter reflect a natural loss of stability of the AP pattern due to spontaneous, self-organizing processes with little or no intentional intervention. It is worth commenting that Aramaki et al. (2006) and others (Debaere et al., 2004) have reported BG activity during the epoch preceding spontaneous pattern transitions, whereas we found no correlation of BG with pattern, rate, or pattern stability before the epoch during which intentional switching occurs. If it is correct to hypothesize that BG activity is modulated by the intrinsic coordination dynamics, in particular by pattern stability, the findings by Aramaki et al. (2006) and Debaere et al. (2004) might be explained by recruitment of the BG for the performance of highly unstable patterns preceding spontaneous transitions. Under this hypothesis, BG would intervene for the maintenance of a less stable AP coordination pattern while inhibiting the inherent tendency for selection of the stable IP pattern under frequency stress. When coordinating at slower rates that preclude spontaneous transitions, as in our paradigm, BG activity may not be required to maintain the ongoing pattern, but would still be recruited in a stability-dependent manner when switching between patterns.

Recent models of information processing in the BG, such as that proposed by Gurney and colleagues (Gurney et al., 2001, 2004; Prescott et al., 2002, 2006; Bar-Gad et al., 2003) propose that the striatum compresses cortical information to determine saliency during selection. This approach is in line with that of Coordination Dynamics in which dimension reduction of the coordinating system is accomplished through collective variables and their low-dimensional dynamics (stability, instability, switching, etc.) The next step is to map the dynamics onto neural structures that putatively underlie behavioral coordination (Kelso et al., 1992; Jantzen et al., 2008, 2009). Our findings that putamen activity during switching was reduced when the intended pattern is compatible with, and thus facilitated by, the intrinsic coordination dynamics suggest that at the neural level, dimensionality reduction is accomplished, possibly within pre- 
motor cortex, through the representation of pattern stability (Jantzen et al., 2009). Moreover, processing within the striatum during intentional switching may rely on the representation of stability to exploit the intrinsic stability of coordination and efficiently drive selective stabilization of the desired pattern at the same time as destabilize competing ones.

Previous studies have suggested that BG may be organized on the basis of coordination synergies (Gurney et al., 2001) in discrete, parallel cortical-BG loops (Alexander et al., 1986; Hoover and Strick, 1993; Graybiel et al., 1994) and somatotopic connections (DeLong et al., 1985; Flaherty and Graybiel, 1993). Recent findings suggested that the BG architecture may be suitable to receive limbic, associative, or sensorimotor inputs and to integrate them to generate a measure of relative salience of competitive behaviors. Here, relative salience may be modeled in terms of pattern stability, which is critical for the inhibition/selection process (Gurney et al., 2001). Evidence in support of this hypothesis comes from the implementation of biologically constrained models of BG architecture in the control of mobile robots during action selection under changing sensory and motivational conditions (Prescott et al., 2002, 2006). A similar mechanism could explain how BG act during intentional switching, that is, convergent sensory, motor, and motivational inputs may provide the BG with information about the intrinsic dynamics of the ongoing and target patterns, and therefore about the availability of competitive and alternative actions. This form of dimension reduction would allow for a context-specific and flexibly adaptive means of pattern selection and switching.

\section{References}

Alexander GE, DeLong MR, Strick PL (1986) Parallel organization of functionally segregated circuits linking basal ganglia and cortex. Annu Rev Neurosci 9:357-381.

Aramaki Y, Honda M, Okada T, Sadato N (2006) Neural correlates of the spontaneous phase transition during bimanual coordination. Cereb Cortex 16:1338-1348.

Bar-Gad I, Morris G, Bergman H (2003) Information processing, dimensionality reduction and reinforcement learning in the basal ganglia. Prog Neurobiol 71:439-473.

Binder JR, Rao SM, Hammeke TA, Frost JA, Bandettini PA, Hyde JS (1994) Effect of stimulus rate on signal response during functional magnetic resonance imaging of auditory cortex. Brain Res Cogn Brain Res 2:31-38.

Cools AR (1980) Role of neostriatal dopaminergic activity in sequencing and selecting behavioural strategies. Facilitation of processes involved in selecting the best strategy in a stressful situation. Behav Brain Res 1:361-378.

Cools R, Sheridan M, Jacobs E, D’Esposito M (2007) Impulsive personality predicts dopamine-dependent changes in frontostriatal activity during component processes of working memory. J Neurosci 27:5506-5514.

Cox RW (1996) AFNI: software for analysis and visualization of functional magnetic resonance images. Comput Biomed Res 29:162-173.

Cox RW, Hyde JS (1997) Software tools for analysis and visualization of fMRI data. NMR Biomed 10:171-178.

Daffertshofer A, Peper CE, Beek PJ (2000) Spectral analysis of event-related encephalographic signals. Phys Lett A 266:290-302.

Debaere F, Wenderoth N, Sunaert S, Van Hecke P, Swinnen SP (2004) Cerebellar and premotor function in bimanual coordination: parametric neural responses to spatiotemporal complexity and cycling frequency. Neuroimage 21:1416-1427.

DeLong MR, Crutcher MD, Georgopoulos AP (1985) Primate globus pallidus and subthalamic nucleus: functional organization. J Neurophysiol 53:530-543.

Flaherty AW, Graybiel AM (1993) Two input systems for body representations in the primate striatal matrix: experimental evidence in the squirrel monkey. J Neurosci 13:1120-1137.

Fuchs A, Deecke L, Kelso JAS (2000a) Phase transitions in human brain revealed by large SQuID arrays. Phys Lett A 266:303-308.

Fuchs A, Mayville JM, Cheyne D, Weinberg H, Deecke L, Kelso JAS (2000b)
Spatiotemporal analysis of neuromagnetic events underlying the emergence of coordinative instabilities. Neuroimage 12:71-84.

Girard B, Filliat D, Meyer JA, Berthoz A, Guillot A (2005) Integration of navigation and action selection functionalities in a computational model of cortico-basal-ganglia-thalamo-cortical loops. Adapt Behav 13:115-130.

Graybiel AM, Aosaki T, Flaherty AW, Kimura M (1994) The basal ganglia and adaptive motor control. Science 265:1826-1831.

Gurney K, Prescott TJ, Redgrave P (2001) A computational model of action selection in the basal ganglia. A new functional anatomy. Biol Cybern 84:401-410.

Gurney K, Prescott TJ, Wickens JR, Redgrave P (2004) Computational models of the basal ganglia: from robots to membranes. Trends Neurosci 27:453-459.

Hoover JE, Strick PL (1993) Multiple output channels in the basal ganglia. Science 259:819-821.

Humphries MD, Gurney K (2000) A computational model of action selection in the basal ganglia: thalamic and cortical interactions. Eur J Neurosci 12 [Suppl 11]:260.10.

Humphries MD, Stewart RD, Gurney KN (2006) A physiologically plausible model of action selection and oscillatory activity in the basal ganglia. J Neurosci 26:12921-12942.

Jantzen KJ, Kelso JAS (2007) Neural coordination dynamics of human sensorimotor behavior: a review. In: Handbook of brain connectivity (Jirsa VK, McIntosh AR eds), pp 421-461. Heidelberg: Springer.

Jantzen KJ, Oullier O, Kelso JAS (2008) Neuroimaging coordination dynamics in the sports sciences. Methods 45:325-335.

Jantzen KJ, Steinberg FL, Kelso JAS (2009) Coordination dynamics of large scale neural circuitry underlying sensorimotor behavior. J Cogn Neurosci 21:2420-2433.

Jenkinson M, Smith SM (2001) A global optimization method for robust affine registration of brain images. Med Image Anal 5:143-156.

Jerbi K, Lachaux JP, N'Diaye K, Pantazis D, Leahy RM, Garnero L, Baillet S (2007) Coherent neural representation of hand speed in humans revealed by MEG imaging. Proc Natl Acad Sci U S A 104:7676-7681.

Kelso JAS (1984) Phase transitions and critical behavior in human bimanual coordination. Am J Physiol Regul Integr Comp Physiol 246: R1000-R1004.

Kelso JAS (1995) Dynamic patterns: the self-organization of brain and behavior. Cambridge, MA: MIT.

Kelso, JAS (2009) Coordination dynamics. In: Encyclopedia of complexity and system science (Meyers RA, ed). Heidelberg: Springer.

Kelso JAS, Scholz JP, Schöner G (1988) Dynamics governs switching among patterns of coordination in biological movement. Phys Lett A 134:8-12.

Kelso JAS, Bressler SL, Buchanan S, DeGuzman GC, Ding M, Fuchs A, Holroyd T (1992) A phase transition in human brain and behavior. Phys Lett A 169:134-144.

Kennerley SW, Diedrichsen J, Hazeltine E, Semjen A, Ivry RB (2002) Callosotomy patients exhibit temporal uncoupling during continuous bimanual movements. Nat Neurosci 5:376-381.

Kraft E, Chen AW, Flaherty AW, Blood AJ, Kwong KK, Jenkins BG (2007) The role of basal ganglia in bimanual coordination. Brain Res 1151:62-73.

Kropotov JD, Etlinger SC (1999) Selection of actions in the basal ganglia thalamocortical circuits: review and model. Int J Psychophysiol 31:197-217.

Lee TD, Blandin Y, Proteau L (1996) Effects of task instructions and oscillation frequency on bimanual coordination. Psychol Res 59:100-106.

Li CS, Yan P, Sinha R, Lee TW (2008) Subcortical processes of motor response inhibition during a stop signal task. Neuroimage 41:1352-1363.

Mayville JM, Bressler SL, Fuchs A, Kelso JAS (1999) Spatiotemporal reorganization of electrical activity in the human brain associated with a timing transition in rhythmic auditory-motor coordination. Exp Brain Res 127:371-381.

Meyer-Lindenberg A, Ziemann U, Hajak G, Cohen L, Berman KF (2002) Transition between dynamical states of differing stability in the human brain. Proc Natl Acad Sci U S A 99:10948-10953.

Mink JW (1996) The basal ganglia: focused selection and inhibition of competing motor programs. Prog Neurobiol 50:381-425.

Park H, Turvey MT (2008) Imperfect symmetry and the elementary coordination law. In: Coordination: neural, behavioral and social dynamics (Fuchs A, Jirsa VK, eds), pp 3-25. Berlin: Springer. 
Paus T (2001) Primate anterior cingulate cortex: where motor control, drive and cognition interface. Nat Rev Neurosci 2:417-424.

Ponzi A (2008) Dynamical model of salience gated working memory, action selection and reinforcement based on basal ganglia and dopamine feedback. Neural Netw 21:322-330.

Prescott TJ, Gurney K, Montes-Gonzales FM, Humphries MD, Redgrave P (2002) The robot basal ganglia: action selection by an embedded model of the basal ganglia. In: Basal ganglia VII (Nicholson L, Faull R, eds), pp 349-356. New York: Plenum.

Prescott TJ, Montes González FM, Gurney K, Humphries MD, Redgrave P (2006) A robot model of the basal ganglia: behavior and intrinsic processing. Neural Netw 19:31-61.

Price C, Wise R, Ramsay S, Friston K, Howard D, Patterson K, Frackowiak R (1992) Regional response differences within the human auditory-cortex when listening to words. Neurosci Lett 146:179-182.

Sadato N, Ibañez V, Deiber MP, Campbell G, Leonardo M, Hallett M (1996) Frequency-dependent changes of regional cerebral blood flow during finger movements. J Cereb Blood Flow Metab 16:23-33.

Scholz JP, Kelso JAS (1990) Intentional switching between patterns of bimanual coordination depends on the intrinsic dynamics of the patterns. J Mot Behav 22:98-124.

Schöner G, Kelso JAS (1988) A dynamic pattern theory of behavioral change. J Theor Biol 135:501-524.

Seger CA (2008) How do the basal ganglia contribute to categorization?
Their roles in generalization, response selection, and learning via feedback. Neurosci Biobehav Rev 32:265-278.

Shima K, Tanji J (2000) Neuronal activity in the supplementary and presupplementary motor areas for temporal organization of multiple movements. J Neurophysiol 84:2148-2160.

Swinnen SP (2002) Intermanual coordination: from behavioural principles to neural-network interactions. Nat Rev Neurosci 3:348-359.

Talairach J, Tournoux P (1988) Co-planar stereotaxic atlas of the human brain: 3-dimensional proportional system-an approach to cerebral imaging. New York: Thieme Medical.

Thickbroom GW, Phillips BA, Morris I, Byrnes ML, Mastaglia FL (1998) Isometric force-related activity in sensorimotor cortex measured with functional MRI. Exp Brain Res 121:59-64.

Toxopeus CM, de Vries PM, de Jong BM, Johnson KA, George MS, Bohning DE, Walker J, Leenders KL (2007) Cerebral activation patterns related to initiation and inhibition of hand movement. Neuroreport 18:1557-1560.

Troyer AK, Black SE, Armilio ML, Moscovitch M (2004) Cognitive and motor functioning in a patient with selective infarction of the left basal ganglia: evidence for decreased non-routine response selection and performance. Neuropsychologia 42:902-911.

Yehene E, Meiran N, Soroker N (2008) Basal ganglia play a unique role in task switching within the frontal-subcortical circuits: evidence from patients with focal lesions. J Cogn Neurosci 20:1079-1093. 